\title{
Transitive Statistical Sensor Error Characterization and Calibration
}

\author{
Jessica Feng \\ Computer Science Department \\ University of California, Los Angeles \\ Los Angeles, California, USA \\ jessicaf@cs.ucla.edu
}

\author{
Miodrag Potkonjak \\ Computer Science Department \\ University of California, Los Angeles \\ Los Angeles, California, USA \\ miodrag@cs.ucla.edu
}

\begin{abstract}
Calibration is the process of identifying and correcting for the systematic bias component of the error in the sensor measurements. On-line and in-field sensor measurement calibration is particularly crucial since manual calibration is expensive and sometimes infeasible. We have developed an on-line and in-field error modeling technique, which is a generalization of the calibration problem, that relies on a small number of inaccurate sensors with known error distributions to develop error models for the deployed in-field sensors. We demonstrate the applicability of our transitive error modeling technique and evaluate its performance in various scenarios by conducting experiments using traces of the light intensity measurements recorded by in-field deployed light sensors. In addition, statistical validation and evaluation methods such as resubsitution are used in order to establish the interval of confidence.
\end{abstract}

\section{INTRODUCTION}

Device imperfections and the natural process of deterioration, along with other sources have contributed to the inevitable errors in sensor measurements. Traditionally, the calibration problem has been defined as identifying and compensating the most likely error value. Measurement error modeling is a generalization of the calibration problem where the goal is not just to find the single most likely error, but also to derive the probability of any error occurring for a given measurement. Consequently, error modeling provides the flexibility of correcting the bias based on other objectives such as the minimum normalized error, besides the traditional most likely error value. We propose an on-line infield error modeling approach that relies on a small number of inaccurate sensors with known (or derived) error distributions to develop error models for the deployed infield sensors.

The approach involves three entities: expensive and accurate instruments - the meters; the deployed sensors uncalibrated sensors; and some number of additional notdeployed uncalibrated sensors whose error distributions can be obtained off-line - the intermediate sensors. The approach has three phases. During the first phase, the error models of the intermediate sensors are constructed by referencing the meters (i.e. the correct values) in a controlled environment (e.g. a laboratory). Then we place the intermediate sensors along sides of the uncalibrated sensors to obtain the error distributions of the uncalibrated sensors with respect to the intermediate sensors readings during the second phase. In the last phase, we transitively merge the two error models to construct the error models for the uncalibrated sensors with respect to the correct values (the meter readings). Note that our transitive calibration approach is economically viable since light meters are considerably more expensive (may cost up to $\$ 1,000$ ) than light sensors (may be purchased for as little as $\$ 0.25$ )

\section{RELATED WORK}

In this Section, we survey the related literature on sensor calibration, especially on-line in-field calibration techniques and statistical modeling and regression techniques.

Calibration in the context of sensor networks has a unique set of requirements and constraints due to the specifics of how sensor networks are deployed and operated. In particular, the needs for low power operation and low bandwidth communication, the fact that harsh environments accelerate the degradation of sensors, and the cost of sensitivity pose unique challenges. Some of the existing state-of-the-art calibration techniques for sensor networks can be found in the References section [1][2][3][4][5][6]. For example, Hightower et al. [2] use the same type of model to fit a combination of linear and logarithmic functions. To the best of our knowledge, no existing efforts has been made on constructing error models using nonparametric statistical methods; nor reporting statistical evaluation of the developed models in terms of interval of confidence.

One of the state-of-the-art on-line and in-field calibration techniques is proposed by Bychkovskiy et al. [1]. The basis for their work is an observation that two sensors that are spatially close often have temporarily correlated recordings. The approach consists of two phases. During the first phase, the goal is attempt to eliminate or reduce the systematic error 
by considering pairs of physically close sensors. During the next phase, they consider a large number of pairs of sensors and try to find the most consistent way to simultaneously satisfy all pair-wise relationships. The approach is fully online and the sensors are calibrated according to the most consistent consensus among all pairs of sensors. We propose a new approach that utilizes accurate meters as the reference to transitively construct error models for the deployed sensors on-line via the intermediate sensors. Therefore, it is most robust against the situation of large number of faulty sensors. In addition, error model also provides the complete probability information of all possible values given a particular uncalibrated sensor's reading.

Least linear-squares fitting/regression is one of the most popular parametric techniques for fitting a set of data to a particular compressed form. The technique is optimal under the assumption that the error distribution follows the Gaussian distribution. The standard technique for conducting least squares fitting is to use the Singular Value Decomposition (SVD). In addition, there are various available techniques that can be applied to determine whether the least squares model is appropriate for the given set of data. Many publications provide the detailed description of these methods [7][8][9]. In addition to parametric techniques, nonparametric techniques that do not depend on any underlying assumptions have been gaining popularity, such as kernel density estimation. Some of the standard references of nonparametric regression techniques can be found in the References section [10][11][12][13].

\section{Transitive SEnsor CALIBRAtion TECHNIQUE}

In this section, we start by formally defining the error modeling and calibration problem. Then we describe the three phases of our transitive approach in detail followed by demonstrating the use of kernel density estimation method for error modeling.

\section{A. Problem Formulation}

A deployed network consists of a large number of uncalibrated sensors. A limited number of additional notdeployed sensors and an accurate instrument are also available in a controlled environment. The goal is to develop error models for the uncalibrated sensors with respect to the accurate values measured by the meter. For the sake of simplicity, we demonstrate the concept of our transitive error modeling technique based on one meter $M$, one intermediate sensor $I$ and one uncalibrated deployed sensor $U$. Let $r_{m}$ denotes a reading from the meter, $r_{i}$ denotes a reading from the intermediate sensor, and $r_{u}$ denotes a reading from the deployed uncalibrated sensor. The goal is to construct an error model of the unclibrated sensor $U$ with the meter readings as the reference values. The error model provides the probability of any reference value (i.e. the meter reading) $P\left(r_{m}\right)$ given any particular $r_{u}$.

\section{B. Error Model Construction}

Our transitive on-line error modeling technique consists of three phases. During the first phase, we place the meter $M$ and the intermemdiate sensor $I$ next to each other in a controlled environment so that they measure the identical physical phenomenon. We utilize pairs of measurements: $r_{m, t}$ and $r_{i, t}, t=1, \ldots, T_{1}$, to construct the probability density function (PDF). The corresponding PDF is shown in Figure 1 , where given a reading of the intermediate sensor, the PDF provides the complete probability distribution of all possible meter readings. During the second phase, we place the intermediate sensor $I$, whose error distribution is obtained in the first phase, next to the deployed uncalibrated sensor $U$. The identical procedure is used to obtain the PDF using the measurements from the intermediate sensors $r_{i, t}$ and the uncalibrated sensors $r_{u, t}, t=1, \ldots, T_{2}$. For the sake of simplicity, let $T=T_{l}=T_{2}$. The PDF is constructed on-line depicted in Figure 2. Both error models (PDFs) are developed using the kernel density estimation technique [14], which is described in the next subsection. During the third phase, the two PDFs obtained in the first two phases are transitively combined as shown in Equation (1) in order to derive the PDF of the uncalibrated sensor with respect to the meter readings. Figure 3 shows the transitively derived PDF.

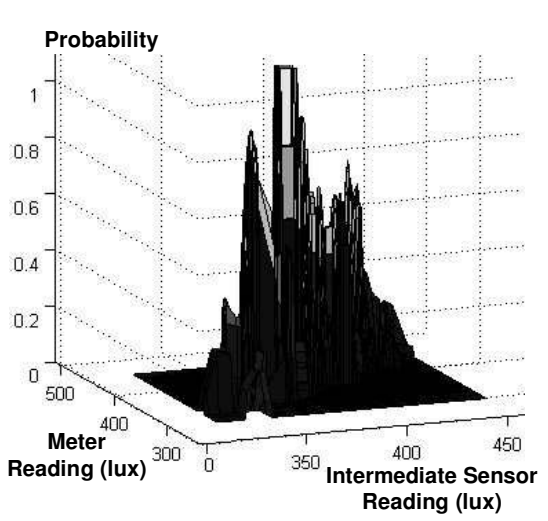

Figure 1. The PDF of the intermediate sensor readings with respect to the meter readings.

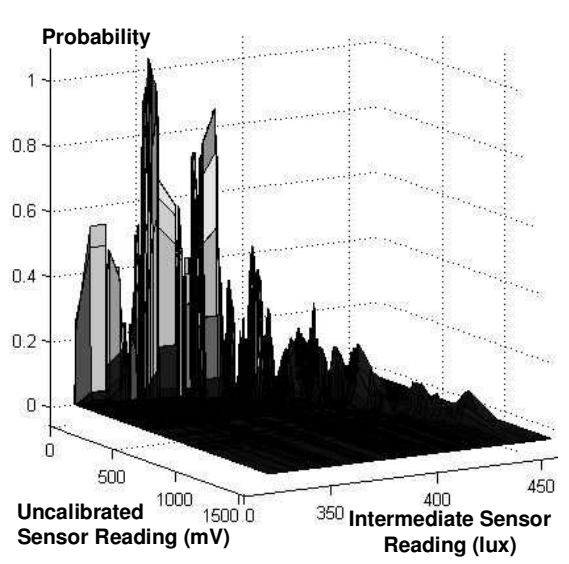

Figure 2. The PDF of the uncalibrated sensor readings with respect to the intermediate sensor readings.

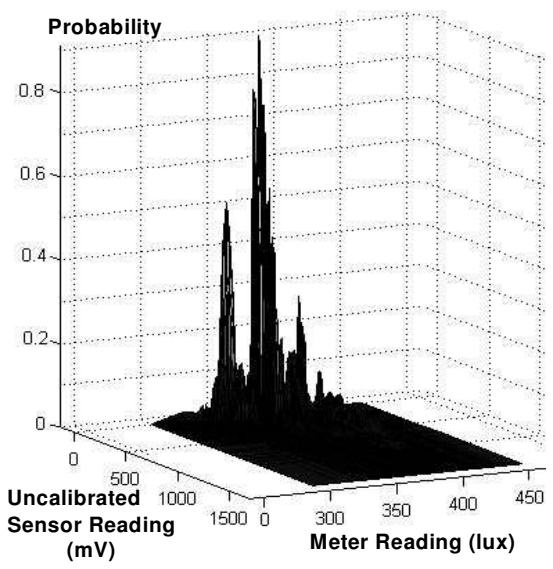

Figure 3. The PDF of the uncalibrated sensor readings with respect to the meter readings. 


$$
P\left(r_{m, t} \mid r_{u, t}\right)=\sum_{l=1}^{T} P\left(r_{m, t} \mid r_{i, t}\right) \cdot P\left(r_{i, l} \mid r_{u, t}\right)
$$

\section{Kernel Density Estimation}

Within the framework of sensor fusion, it is common for the Gaussian distribution model to be assumed and the least linear-squares to be used in order to minimize discrepancy. For linear systems, that approach is optimal under the assumption of Gaussian noise distribution. However, a number of recent experiments indicate that errors in sensor systems do not follow the Gaussian distribution and often have complex behavior that cannot be captured using the standard distributions and parametric statistical methods. Our goal is to construct statistical error models using nonparametric statistical techniques where no assumption on error distribution is required.

The PDFs are constructed using a combination of Nadaraya-Watson kernel-weighted average in conjunction with local linear regression - equivalent kernel [14][15][16]. Such an error model not only provides one single most probable correct value for a given measurement, it also provides the complete probability distribution of all possible correct values for a single given measurement. During the first phase, the measurements are the readings from the intermediate sensor, while the reference (or correct) values are the meter's readings. During the second phase, the measurements are the readings from the in-field uncalibrated sensor, while the reference values are the readings from the intermedate sensor. The PDF is constructed by examining the frequency of different reference values given a single measured value. However, the most common difficulty towards constructing an accurate statistical error model is the lack of sufficient number of measurement data. To address this limitation, the sliding window kernel density estimation technique [14][17] is used to construct the PDF, which is a function of two variables: the intensity of the measurements and the corresponding reference values.

The Nadaraya-Watson kernel-weighted average uses $k$ nearest neighbors of $x$ in order to compute the expected (or

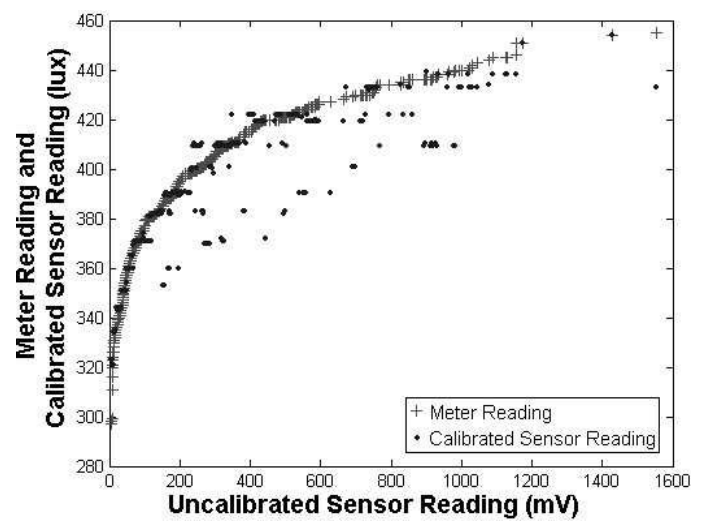

Figure 4: The comparison between the meter readings (correct values) and the calibrated values (using the transitive technique) given the uncalibrated sensor readings. the most likely) value of $x$. In addition, rather than assigning all $k$ points in the neighborhood equal weight, we assign weights that die off smoothly with distance from the target point $x$. It is well known that locally weighted averages can be heavily biased on the boundaries of the domain because of the asymmetry of the kernel in those regions. By conducting a separate weighted least squares linear regression, and combining the weighting kernel and the least squares operations, the bias can be removed exactly to the first order.

Consider a set of $n$ pairs of measured and reference values $\left\{\left(x_{i}, y_{i}\right), i=1, \ldots, n\right\}$. For each measurement $X$, we define a neighborhood (window) of size $X \pm\left(\Delta \varepsilon_{x}\right)$, and consider all the measurements within this window to construct a 2-dimensional PDF curve of the reference values for $X$. Furthermore, this 2-d curve is constructed by applying the same technique on the reference values. For each reference value $Y$, we define another window of size $Y \pm\left(\Delta \varepsilon_{y}\right)$, all reference values fall within both of these two windows (i.e. $\left.\left\{\left(x_{i}, y_{i}\right) \mid x_{i} \in X \pm\left(\Delta \varepsilon_{x}\right), y_{i} \in Y \pm\left(\Delta \varepsilon_{y}\right)\right\}\right)$ are used to estimate the probability of $Y$ given the $X$. Note that the window sizes ( $\Delta \varepsilon_{x}$ and $\Delta \varepsilon_{y}$ ) determine the width of the local neighborhood. Large window size yields lower variance (average over more data points) and thus less specificity for each individual error. After computing the kernel weights of all neighboring points, the least linear squares regression is then imposed on the weighted data points.

\section{EXPERIMENTAL RESULTS}

In this section, we present and analyze the experimental results obtained by applying our transitive calibration technique on a set of deployed light sensors. We evaluate the transitive error modeling technique in three aspects: i) calibration error, defined as the ratio between the discrepancy of the calibrated sensor reading according to the error model compared to the meter reading and the meter reading; ii) interval of confidence, and iii) how calibration accuracy degrades as the level of transitivity increases.

Once the error models are constructed, the calibration function used to map the recorded values to the referencing

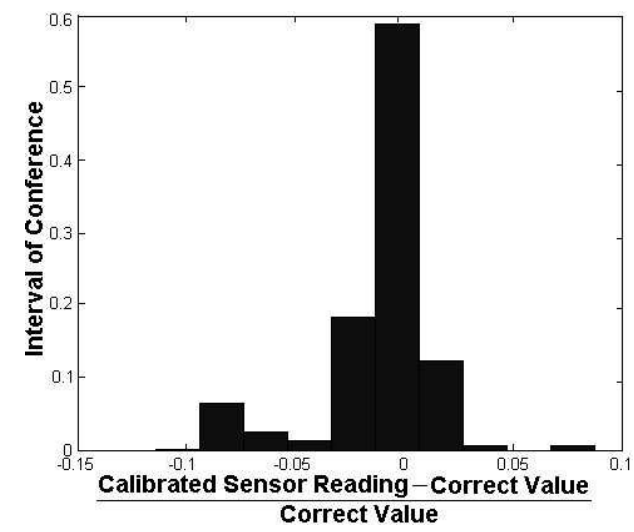

Figure 5: The interval of confidence of the calibration error. The correct values are the meter readings. 
values in order to compensate for sensor bias can be defined in different ways. In Figure 4, we show the calibrated values selected based on the maximal likelihood principle in comparison to the meter readings for an arbitrarily selected uncalibrated sensor. One of the main side benefits of using nonparametric statistical techniques for calibration is that in addition to calibration mapping, we are also able to derive the interval of confidence for either a particular measurement value or for all measurement values overall. The interval of confidence is derived using the standard resubstitution method, where $m \%$ of the original data is randomly selected to construct the error model, and the remaining $(1-m) \%$ of the data is used to evaluate the model. This process is repeated $P$ times to construct the interval of confidence. $P$ is set to 200 in our experiments. For example, Figure 5 shows the interval of confidence in terms of histograms of the calibration errors shown in Figure 4 when $m=70 \%$ of the training data is given. We can conclude from the figure that with $90 \%$ of confidence, an average of $0 \pm 2.5 \%$ of the calibration error is consistently achieved.

In addition, we studied how the calibration accuracy degrades as the levels of intermediate sensors increases. Figure 6 shows the calibration errors in terms of boxplots of the same arbitrarily selected unclibrated sensor. The top and the bottom whiskers of the boxplot are the maximum and the minimum of the calibration errors without considering $25 \%$ of the outliers beyond the whiskers; the upper line of the box, the line in the box, and the bottom line of the box are the $75 \%, 50 \%$ (mean), and the $25 \%$ percentiles of the calibration errors, respectively. We can conclude that even with 5 levels of intermediate sensors, $75 \%$ of the readings of the uncalibrated sensor still can be calibrated with a maximum error of $11.2 \%$.

In principle, as the title of the paper implies, our new calibration approach can be used as a generic method for the regression task of predicting a target variable that is difficult to deduce from an explanatory variable that is easy to measure. However, it is important to emphasize that the effectiveness of all statistical techniques greatly depends on the nature and the properties of the analyzed data set. Therefore, while our statistical methods perform well as a calibration tool, it may or may not be suitable for other arbitrary given regression tasks.

\section{CONCLUSION}

We propose a new transitive on-line and in-field error modeling and sensor calibration technique. The approach utilizes a small number of accurate and expensive meters and a limited number of inexpensive and uncalibrated intermediate sensors to transitively construct error models for the deployed sensors. The error models allow the calibration function to be defined according to different objectives. In addition to calibration accuracy and confidence level, we also quantitatively studied the applicability of multi-level intermediate sensors.

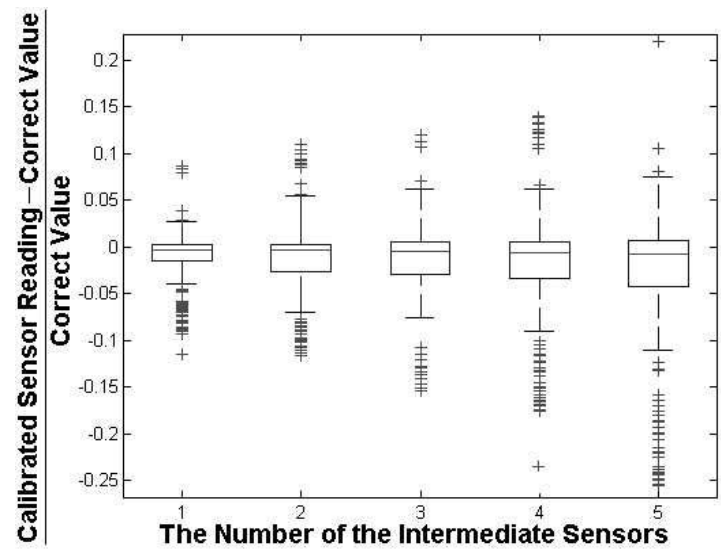

Figure 6. The boxplots of the calibration error given different numbers of the transitive intermediate sensors.

\section{REFERENCES}

[1] V. Bychkovskiy, S. Megerian, D. Estrin, and M. Potkonjak, "Colibration: a collaborative approach in-place sensor calibration," International Workshop on Information Processing in Sensor Networks, 2003, pp. 301-316.

[2] J. Hightower, C. Vakili, and G. Borriello, "Design and calibration of the SpotON ad-hoc location sensing system," UW CSE, University of Washington, August 2001.

[3] A.T. Ihler, and J.W. Fisher, "Nonparametric belief propagation for self-calibration in sensor networks," International Workshop on Information Processing in Sensor Networks, 2004, pp. 225-233.

[4] A. LaMarca, W. Brunette, D. Koizumi, M. Lease, S.B. Sigurdsson, K. Sikorski, D. Fox, and G. Borriello, "Making sensor networks practical with robots," IEEE International Conference on Pervasive Computing and Communications, 2002.

[5] J.Feng, G. Qu, and M. Potkonjak, "Sensor Calibration Using Nonparametric Statistical Characterization of Error Models" IEEE Conference on Sensors, 2004.

[6] K. Whitehouse, and D. Culler, "Calibration as parameter estimation in sensor networks," ACM International Workshop on Wireless Sensor Networks and Application, 2002, pp.59-67.

[7] D.A. Belsley, E. Kuh, and R.E. Welsch, Regression Diagnostics, Wiley, New York, 1980.

[8] S. Chatterjee, and B. Price, Regression Analysis by Example, John Wiley, New York, 1977.

[9] P.I. Good, Resampling Methods, Springer-Verlag, New York, NY, 1999.

[10] A.C. Davison, and D.V. Hinkley, Bootstrap Methods and their Application. Cambridge University Press, Cambridge, UK, 1997.

[11] B. Efron, The Jackknife, the Bootstrap, and Other Resampling Plans, Society for Industrial \& Applied Mathematics, 1982.

[12] B. Efron, and R. Tibshirani, An Introduction to the Bootstrap, Chapman-Hall, London, 1993.

[13] D. Kahaner, C. Moler, and S. Nash, Numerical Methods and Software. Prentice Hall, Englewood Cli.s, New Jersey, 1989.

[14] T. Hastie, R. Tibshirani, and J. Friedman, The Elements of Statistical Learning: Data Mining, Inference, and Prediction, Springer-Verlag. New York. 2001.

[15] J. Fan, and I. Gijbels, Local Polynomial Modeling and Its Applications, Chapman and Hall, London, 1996.

[16] R. Thisted, Elements of Statistical Computing: Numerical Computation, CRC Press, 1988.

[17] P. Green, and B. Silverman, Nonparametric Regression and Generalized Linear Models: A Roughness Penalty Approach, Chapman and Hall, London, 1994. 